EPJ Web of Conferences 92,02083 (2015)

DOI: $10.1051 /$ epjconf/ 20159202083

(C) Owned by the authors, published by EDP Sciences, 2015

\title{
Aeration equipment for small depths
}

\author{
Jan Sluše ${ }^{1, a}$, František Pochylý ${ }^{1}$ \\ ${ }^{I}$ Brno University of Technology, Faculty of Mechanical Engineering, Victor Kaplan Department of Fluids Engineering, \\ Technická 2, 61669 Brno, Czech Republic
}

\begin{abstract}
Deficit of air in water causes complications with cyanobacteria mainly in the summer months. Cyanobacteria is a bacteria that produces poison called cyanotoxin. When the concentration of cyanobacteria increases, the phenomena ,algal bloom“ appears, which is very toxic and may kill all the organisms. This article describes new equipment for aeration of water in dams, ponds and reservoirs with small depth. This equipment is mobile and it is able to work without any human factor because its control is provided by a GPS module. The main part of this equipment consists of a floating pump which pumps water from the surface. Another important part of this equipment is an aerator where water and air are blended. Final aeration process runs in the nozzles which provide movement of all this equipment and aeration of the water. Simulations of the flow are solved by multiphase flow with diffusion in open source program called OpenFOAM. Results will be verified by an experiment.
\end{abstract}

\section{Introduction}

The quality of water in dams, ponds and reservoirs is being affected by many factors. The main factors influencing quality of water and processes in the water is a concentration of oxygen and solar irradiance which affects the temperature inside the water reservoir. The oxygen is essentially important for the metabolism of all aerobic water organism. The consumption of dissolved oxygen in water is not performed only by aerobic organism but it is used for oxidation processes on the interface of water and sediment. Then the sediment is used as a fertile soil for creation of Cyanobacteria which start to proliferate when aerobic condition changes to anaerobic. The concentration of oxygen in dams is closed related with temperature in the dam. Figure 1 shows the dependence of solubility of oxygen in the water on temperature. In case of increase of temperature of water the solubility of oxygen decreases and the oxygen is released respectively is absorbed in lower amount. The temperature of water in the dam changes with the changes of solar radiance throughout the year. The terms spring, summer, autumn and winter stratification have been established. Each of these terms represents typical structure of temperature layers in the water dam.

Stability is typical for temperature stratificated dam. Stability is defined as amount of work or mechanical energy need to mix the entire volume on the same level of temperature. This process takes place on selected area without any added or lost heat.

The spring stratification is probably the worst type of stratification due to dependence of solubility of the oxygen on temperature (Figure 2) because deficit of oxygen in the water occurs very often (Figure 3 ).

In the nature the stratific layers are interfered by wind, rain, living organisms and another external influence. Such destratification occurs mainly in epilimnion. Natural destratification occurs only sometimes. There is a lack of natural destratification in hot summer months and the overgrowth of cyanobacteria may occur.

Cyanobactera is a bacteria that produces poison called cyanotoxin. When the concentration of cyanobacteria increase, the phenomena ,algal bloom" appears, which is very toxic and may kill all the organisms. [1] [2] [3]

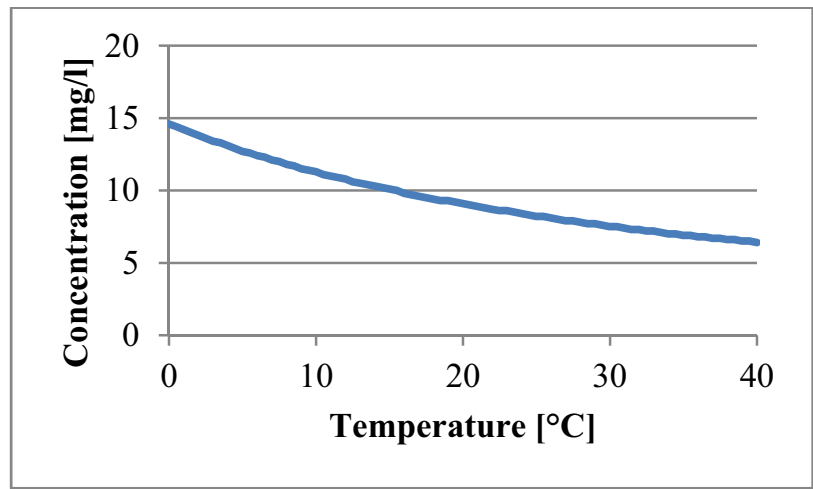

Figure 1. Dependence of the solubility of air at a temperature.

${ }^{\mathrm{a}}$ Corresponding author: y107565@stud.fme.vutbr.cz 


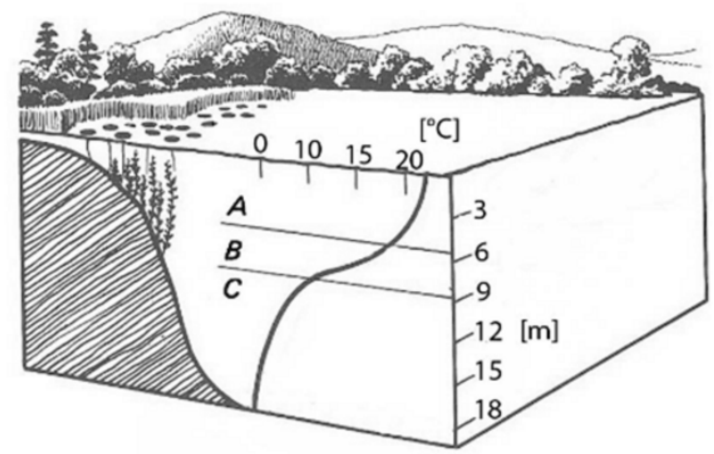

Figure 2. Graphic representation of summer stratification with each layers a) epilimnion b) metalimnion c) hypolimnion.

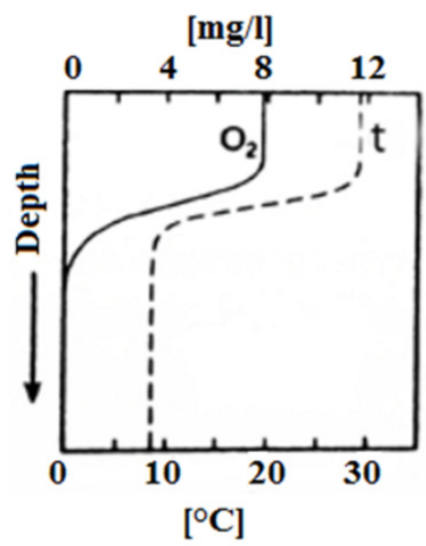

Figure 3. Dependence of oxygen concentration on depth in eutrophic lake; dependence of temperature on depth in eutropic lake.

There are used two ecologic methods to combating Cyanobacteria overgrowth - artificial destratification and aeration. These methods serve as a correction and managing of processes in the water dams. These methods are divided according the place of action onto spotted and areal. The performance of equipment is limited by performance of source of energy, i.e. equipment powered by petrol engine, wind turbine or solar panel.

This article describes new equipment for aeration of water in dams, ponds and reservoirs with small depth. Developed equipment can be used only for a destratification or for destratification combined with aeration.

\section{Proposal of aerator}

Equipment is designed as a mobile aeration and destratification unit which can be used in standing water with small depth (Figure 4). Power is provided by a petrol engine which is connected with high pressure pump. Oxygenated water is pumped across perforated inlet element from water surface where the water is oxygenated the most. Perforated element is used to prevent inflow bigger impurities into pump which my destroy it. In the next step the water is transported from pump to the aerator. This equipment utilizes two types of aeration elements. First is ejector and second is plate aerator (difference is explained below).Water is subsequently pumped into a distribution element which is composed of six electric valves. Operation of these valves is provided by an operation unit next to the pump. Oxygenated water flows into distribution elements which ensure distribution of oxygenated water into lower layers. Aerated water with lower density goes up and mixes with non-aerated water above. The whole equipment is positioned on the floating element. The process of destratification or aeration with destratification might be realized continuously.

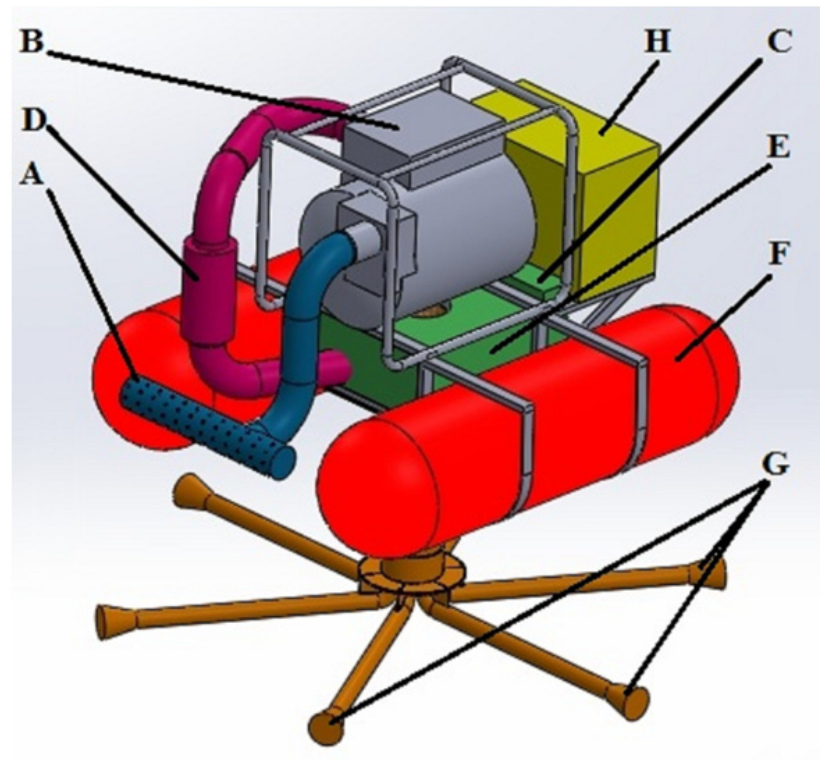

Figure 4. Graphic proposal of aerator.

The legend of the elements (see figure 4) is the following: A - perforated inlet element, B - pump with petrol engine, $\mathrm{C}$ - valves, $\mathrm{D}$ - ejector, $\mathrm{E}$ - plate aerator, $\mathrm{F}$ float, $\mathrm{G}$ - distribution elements, $\mathrm{H}$ - control unit.

\subsection{Pump}

Pump consist of a petrol engine with power of $4.4 \mathrm{~kW} / 3600 \mathrm{rpm}$. Maximal delivery head is $90 \mathrm{~m}$ and maximal delivery volume is $500 \mathrm{l} / \mathrm{min}$. Pump characteristic is shown on Figure 5.

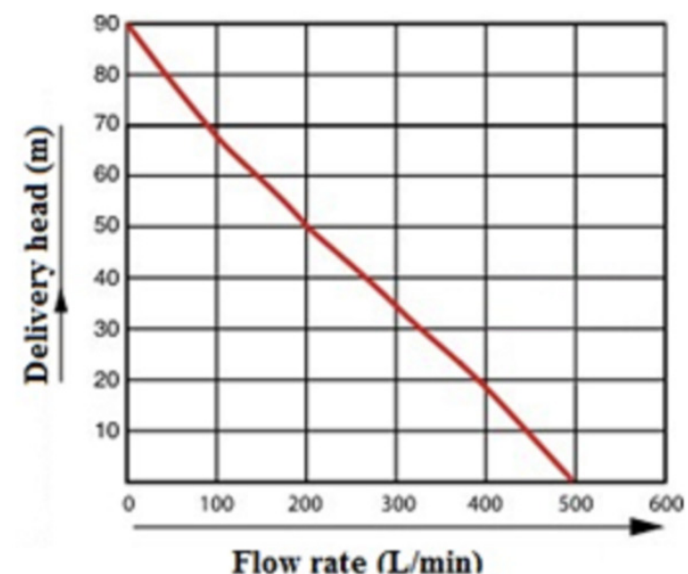

Figure 5. Pump characteristics. 


\subsection{Aeration by ejector}

In the inlet section of the ejector are the distribution vanes which ensure rotation of the liquid. Then the liquid flows through the narrow area of the neck where pressure energy changes into kinetic energy. It is followed by a mixing chamber where due to low pressure and high velocity the air is drawn in and mixed with the liquid. Air is drawn through the perforated sheet which serves as a microbubble generator. Subsequent extension of the shape reduces velocity of the mixture and increases pressure. As a result the diffusion coefficient increases and the velocity of diffusion rises.

\subsection{Plate aerator}

The plate aerator consists of the system of thin metallic boxes provided with porous material upper edge. Air is supplied from the compressor into these boxes.

Due to the special hydrophobic layer the air is held on the perforated surface (due to surface tension) and diffusion occurs. If the air was not held by surface forces it would only bubble through water and the energy performance of the process would be too high.

\subsection{Control unit}

Control unit contains an operation system with GPS module and compass. Both GPS module and compass detect a current position of the equipment. This information is transmitted into the control unit. In the control unit data are compared with the map date of the dam and with data obtained by measuring of oxygen concentration. Measurement of oxygen concentration is provided by an oxygen probes during the whole activity of the equipment. On the basis of these values the operation unit selects an optimal way of move of the equipment and gives impulse to open or close the valves. Only three of all these six valves can be opened at the same time. After a predefined period the operation unit provides a feedback and compares a current position with calculated position. In case of difference of both positions the correction is provided.

\subsection{Distribution elements}

Distribution elements are moved by $60^{\circ}$ to each other. These elements ensure the distribution of modified water into deeper layers of the water column and propulsion of whole unit. Maximum three distribution elements can be in operation.

\section{Basic facts about oxygen diffusion into water}

\subsection{Henry's law [4]}

Henry's law generally applies to solubility of a gas in a liquid. It states: At a constant temperature, the amount of a given gas that dissolves in a given type and volume of liquid is directly proportional to the partial pressure of that gas in equilibrium with that liquid. This law is very important when using aeration by air, not by oxygen, because it specifies the parameter of solubility of individual gas in a liquid.

$$
C_{i}=K_{i} \cdot P_{i}
$$

where $\mathrm{C}_{\mathrm{i}}-$ concentration of gas in solution, $\mathrm{K}_{\mathrm{i}}-$ coefficient of absorption, $\mathrm{P}_{\mathrm{i}}-$ partial pressure of gas above the solution, $\mathrm{i}$ - index which marks gas.

\subsection{First Fick's law [4]}

Solute will move from a region of high concentration to a area of low concentration across a concentration gradient.

$$
J=-D \frac{\partial \Phi}{\partial x}
$$

where $\mathrm{J}$ - diffusion flux, D - diffusion coefficient, $\Phi-$ concentration in dimensions, $\mathrm{x}-$ position.

\subsection{Second Fick's law [4]}

Second Fick's law predicts how diffusion causes the concentration to change with time.

$$
\frac{\partial \Phi}{\partial t}=D \frac{\partial^{2} \Phi}{\partial x^{2}}
$$

where $\mathrm{t}$ - time

\section{Simulations}

Drawings were created in Creo Elements Direct Modeling Express 4.0. This software is free. This software enables to export the geometry in STL format and to set various parameters of the STL conversion in order to achieve a required quality of the geometry. The STL file can be used for mesh generation with snappyHexMesh utility within OpenFoam software.

For the CFD simulations OpenFoam software was used. It is free opensource software for $3 \mathrm{D}$ numerical analysis of fluid flow problems. It contains solvers for Navier Stokes equations coupled to equations of variety of turbulence models. The flow of gas or liquid can be solved in laminar or turbulent regime, steady or unsteady, compressible or incompressible. The user can choose from several algorithms the one which fits best to the physical problem. The OpenFoam package also contains a Paraview software for postprocessing the computed data.

\subsection{Mesh and simulations settings}

For generation of a computational mesh the snappyHexMesh utility of OpenFoam was used. The mesh contains approx. 1.6 millions of cells which are mostly hexahedral. Typical size of cells is $1 \mathrm{~mm}$. 
The overall mesh is depicted in Figure 6. The Figure 7 represents a detail of mesh closed an aeration module.

The numerical problem was solved as a laminar, nonstationary, incompressible flow with twoLiquidMixingFoam solver with diffusion.

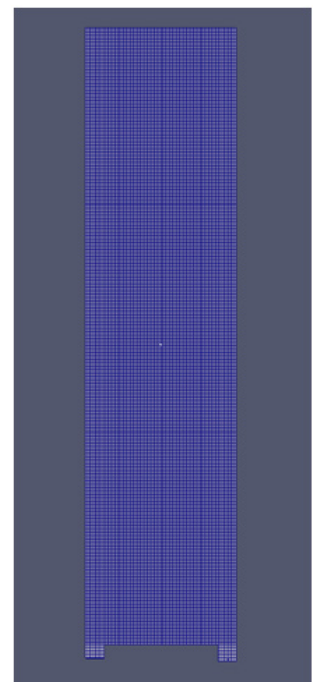

Figure 6. The mesh - complete view.

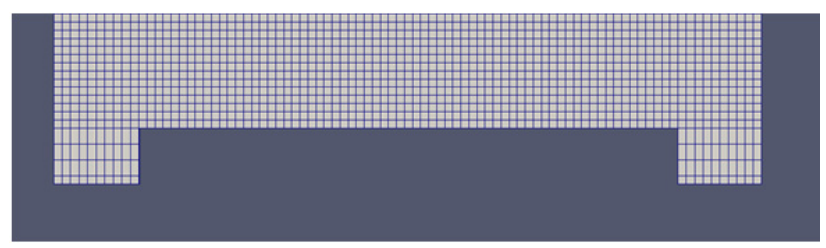

Figure 7. Detail of a mesh closed an aeration module.

\subsection{Results}

Figures 8 and 9 show the distribution of particular phases. Value 1 is for fully oxygenated water under certain conditions (pressure, teperature). Value 0 is for water without oxygen. Figure 10 represents evolution of oxygen content over time.

\section{Experiments}

\subsection{Experimental setup}

The basis for the experimental equipment is an aeration module which is positioned in the glass tube. The aeration modul consists of hydrophobic surface applied by a special technology on a porus material fixed to the base. Compressed air enters the bottom side of the porus material at a suitable pressure to keep the hydrophobic surface stable to avoid emergence of bubbles. To meet this condition it is necessary to keep the difference between air pressure and pressure of liquid. The value of pressure difference is maintained by differential pressure gauge or manually. The value of pressure difference depends on the quality of hydrophobic surface and the used matrix. Since the diffusion occurs, it is necessary to bring the air within certain difference continuously into the aeration element. This ensures both a regulation valve and a compressor equiped with pneumatic accumulator. The prcess of aeration is monitored continuously by a concentration probe positioned of approximately in the middle of the glas tube. The probe identifies the amount of air dissolved in the water and the temperature. These values are under the sampling frequency transmitted into the evaluation unit which storage and display them.

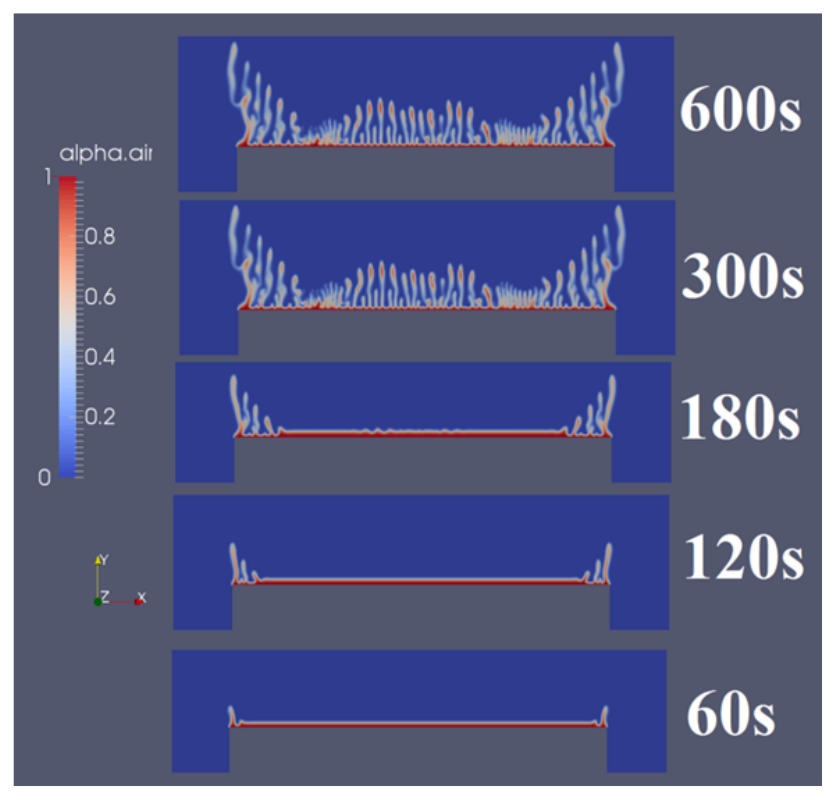

Figure 8. Evolution of particular phases closed to aeration module - the values of time are in seconds.

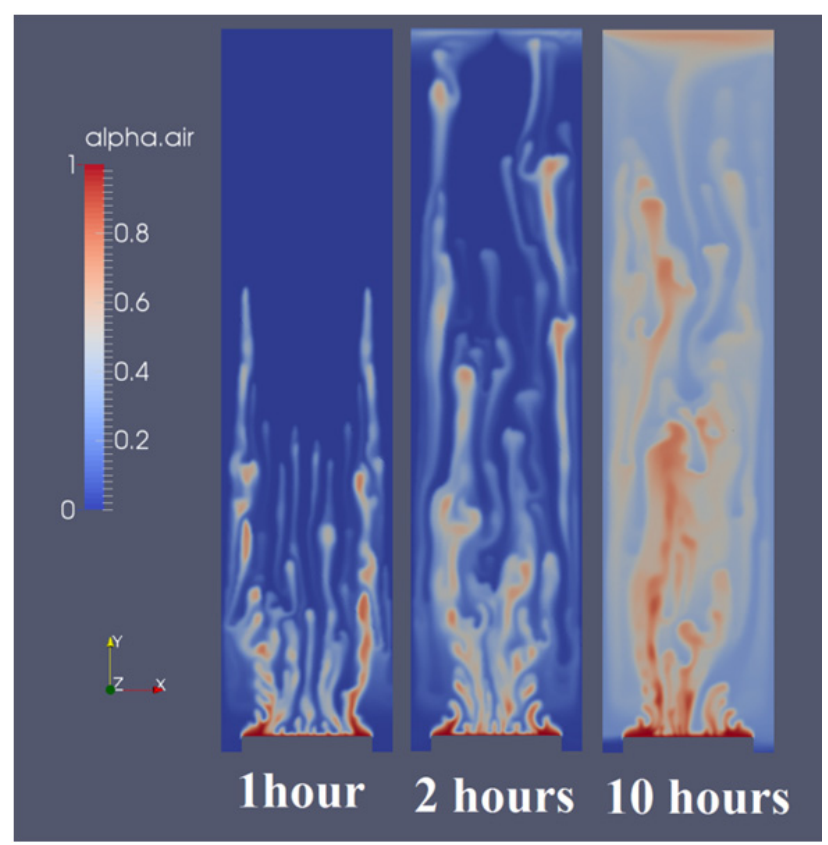

Figure 9. Evolution of particular phases - the values of time are in hours. 


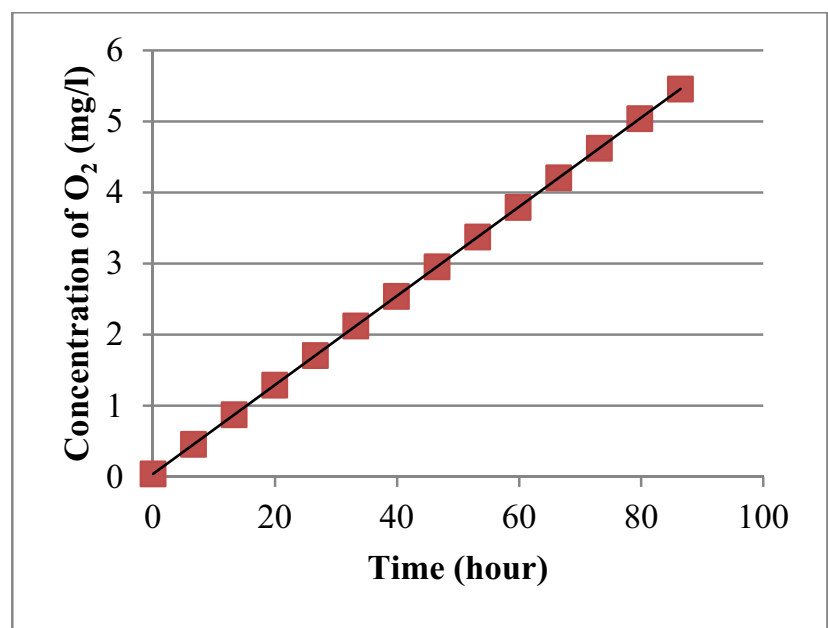

Figure 10. Dependence of the concentration $\mathrm{O}_{2}$ in the water on the time.

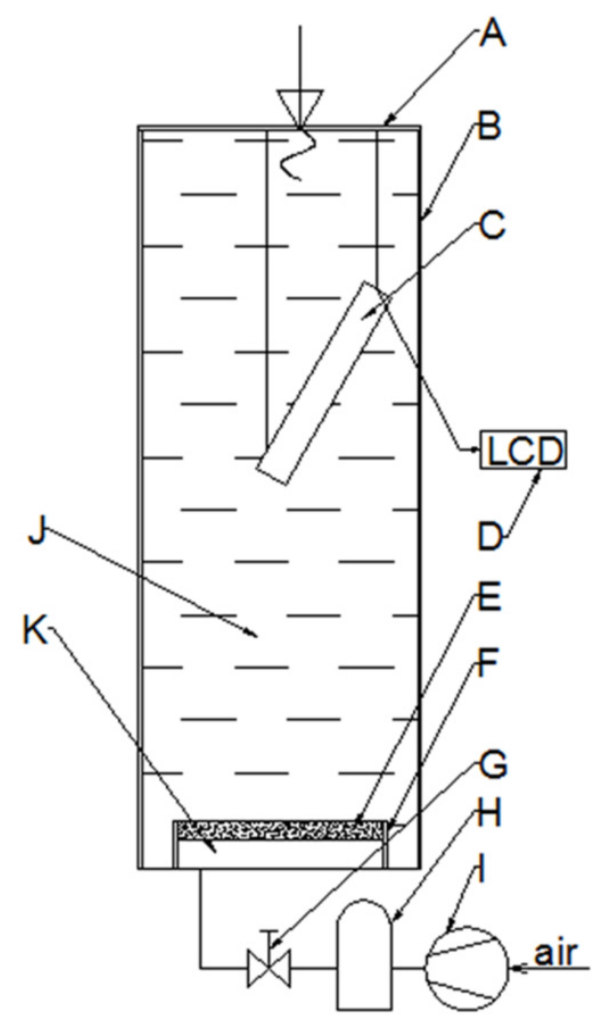

Figure 11. Block diagram of the measuring chain.

The legend of the elements (see figure 4) is the following: $\mathrm{A}$ - cover, $\mathrm{B}$ - glass tube, $\mathrm{C}$ - oxygenation probe, $\mathrm{D}-$ evaluation unit, $\mathrm{E}$ - porous material, $\mathrm{F}$ - base, $\mathrm{G}$ regulatory valve, $\mathrm{H}$ - pneumatic accumulator, I compressor, $\mathrm{J}$ - aerated water, $\mathrm{K}$ - air.

\subsection{The course of the experiment}

On the bottome of glass tube the aeration module is installed and the whole tube is filled with the water. The tube is filled with the tap water and the liquied is allowed to stand for couple of days before use. For the determination of the air content in the water the oxygenation probe is installed. This oxygenation probe is positioned approximatelly in the middle of tube, both horizontally and vertically. The measurment starts after the start up of all control systems and adjustment of the pressure difference.

\subsection{Results}

Due to the measurement the dependence of the concentration $\mathrm{O} 2$ in the water on the time was obtained. On the basis of theoretical knowledges reffered to in previous chapter the solubility of air under certain conditions was calculated. The default value was a value of hydrostatic pressure.

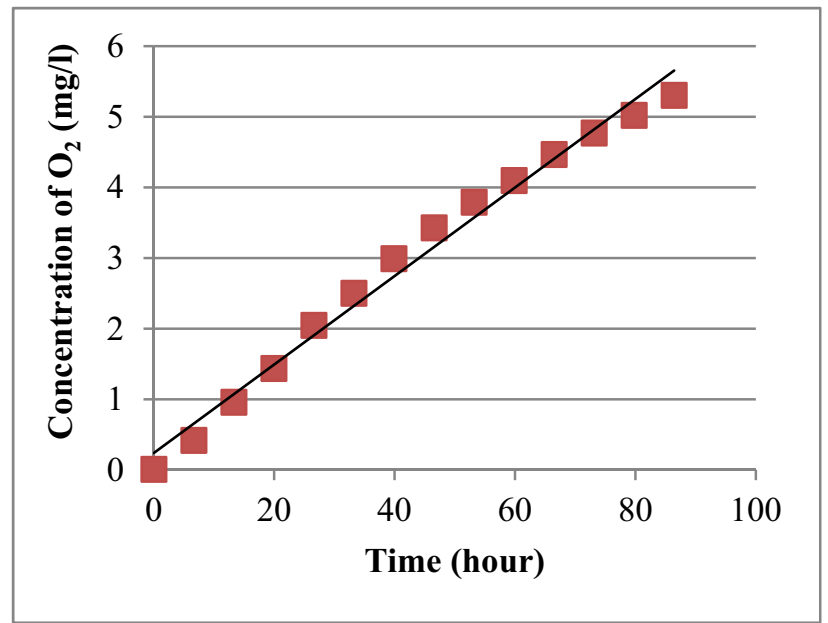

Figure 12. Dependence of the concentration $\mathrm{O}_{2}$ in the water on the time.

\section{Conclusions}

Experimental data were used for a verification of mathematical model used for a simulation. After the comparison it can be stated that the data of simulation are very similar to the experimental data due to appropriately selected diffusion coefficient. The mathematical model can be used for other calculations but it is necessary to keep the conditions of verification. Another essential condition is laminar flow. This mathematic model can be used for calculation of desk ejector where laminar flow is assured. In case of simulation of flow in ejector it is needed to create a test case which is designed for turbulent flow and contains diffusion coefficient for turbulent flow.

It can be seen from the simulation that when designing a desk ejector it is better to choose such position of aeration module that oxygenated water flows upwards against direction of gravity acceleration. Aerated liquid with lower density goes up and mix with the water above. In this case aerated liquid is faster dispersed throughout the volume and high concentration gradient will be kept close to aeration area. This is apparent from CFD simulation. 


\section{Acknowledgments}

The author gratefully thanks to the support of the Czech Science Foundation GA13-20031S.

\section{References}

1. R. Wetzel, G. Limnology, Lake and river ecosystems (Academic Press, San Diego, 2001)

2. P. Pitter, Hydrochemie (VŠCHT, Praha, 2009)

3. R. Bertoni, Limnology of rivers and lakes (Eolss Publishers, Oxford, 2011)

4. F. Incropera, Principles of heat and mass transfer (John Wiley \& Sons, Singapore, 2013) 